\title{
Research Early Mechanical Failure of CNC Motorized Spindle Prediction Method Base on D-S Evidence Theory Information Fusion
}

\author{
Chunyu Mao, a, Guangwen Zhou, ${ }^{1, b}$ and Mei Tian ${ }^{1, c}$ \\ ${ }^{1}$ jilin Enjineerinfg Normal University, Changchun, China

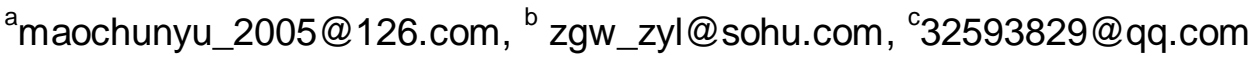

Keywords: Fault diagnosis; FNN; D-S evidence theory; CNC; Motorized spindle

\begin{abstract}
Early mechanical failure of CNC milling motorized spindle having a hidden and complexity is difficult to quickly and accurately identify early machines failure of CNC milling. In this paper, early latent subtle abnormal vibration signals of motorized spindle is detected by PeakVue. Fuzzy neural network diagnosis for each partial signal, and then the sub-diagnosis as evidence, the use of D-S evidence theory to the global final diagnosis, and further improve the early fault recognition rate. Using method of rough set theory data mining obtain processing parts of the surface roughness characteristics of data, which have established the surface roughness of the spectral characteristics of the database. Subsystems, we use FNN fault diagnosis, then the sub-diagnosis as evidence, we use the D-S evidence theory to the global final diagnosis, and further improve the early fault recognition rate. The results show that: This early fault diagnosis model fuzzy neural network and data fusion technology, which is the electrical mechanical failure early prediction accuracy spindle higher generalization ability.
\end{abstract}

\section{Introduction}

CNC milling machine spindle is the main part of the production process, it was found CNC milling spindle has a mechanical problem misdiagnosed, long maintenance cycle and other issues in production. To be able to find fault in advance to avoid large spindle failure, the paper motorized spindle CNC milling machine for the study of milling vibration, noise, temperature, etc. were detected by detecting various signals and trends to predict, can grasp the operational status of equipment and we found that the variation, predict the likelihood of early failure of the spindle to prevent timely detection of abnormal operation signal, so that timely adjustments $[1,2]$.

\section{Method of Signal Acquisition}

Micro Vibration Signal Acquisition. When CNC milling spindle because early mechanical failure, various signals relatively weak, which gives the signal acquisition and processing has brought a lot of difficulties. PeakVue is a new diagnostic technique for rolling bearings and gear fault diagnosis developed vibration signal analysis method for capturing a given time interval in the time-domain waveform peak. Applications PeakVue of technology can find abnormal mechanical vibration signal, especially in the early potential and subtle abnormal signal. When the metal-to-metal collision occurs, it will produce stress waves. Early spalling fatigue, gear and bearing defects, friction and wear and shock wave will cause stress. PeakVue that is through the collection and monitoring of these transient stress waves, and get a peak period occurs and convert the spectral analysis [3, 4].

Surface Roughness Information Collection. Rough set theory is an effective treatment is inconsistent, incomplete data mining information, has the advantage of handling massive data reduction aspects. In addition, the rough set model using only the information provided by the data itself, without modeling to design the model structure and set the model parameters, it has been used to predict the number of complex, large-scale uncertain systems [5, 6]. 


\section{Electric Spindle Early Fault Diagnosis Model D -S Evidence Theory Fusion}

Structure of FNN. FNN is a fuzzy theory and neural networks combine the two products. Nature, people are accustomed to using fuzzy information in logical thinking, judgment and reasoning. Fuzzy theory is on this basis, through the membership function with serial and parallel rules for fuzzy information processing. It has been proved theoretically: fuzzy logic system is able to arbitrary precision approximation of a nonlinear function; and the neural network with nonlinear mapping ability, has a close link between the two. Structure Fuzzy Neural Network consists of three functional modules, one fuzzy module, the input signal is fuzzy processing, introducing membership functions that enable more accurate training sample failure; the second is the ANN learning inference module, which uses artificial neural network with a certain algorithm for CNC machine tool spindle servo system fault diagnosis; the third is clear module, which ultimately determine the cause of the failure based on membership artificial neural network output vector to complete the neural network output mode to the diagnostic results clear process, fuzzy neural network structure shown in Fig. 1[5,6].
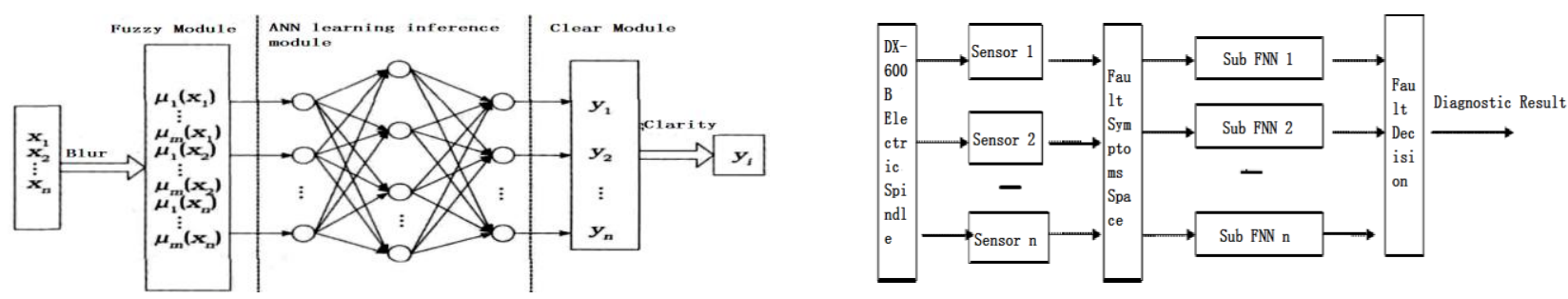

Figure 1. Structure of FNN

Figure 2. FNN Fault Diagnosis Based on Information Fusion Framework

Model of Fault Diagnosis. In this paper, based on the decision-making level information DS evidence theory fusion diagnosis model includes data processing, feature local diagnosis and decision-level fusion of three modules, the structure shown in Fig. 2[6].

D-S Evidence Theory The basic strategy is to evidence collection is divided into several parts that are irrelevant, and were using them to identify the framework for independent judgment, and then use a combination of rules to combine them. Dualistic situation as an example, assume that $\mathrm{Bel}_{1} \mathrm{Bel}_{2}$ and confidence are two functions on the same frame of discernment $\Theta$, where $\Theta$ is the sample space, that is the power set of the set of propositions made a statement incompatible set $2^{\Theta}$ configuration. $\mathrm{m}_{1}$ and $\mathrm{m}_{2}$ corresponding basic probability assignment function, $\mathrm{m} 1$ and $\mathrm{m} 2$ are the focal elements $A_{1}, A_{2}, \ldots, A_{n}$ and $\mathrm{B}_{1}, \mathrm{~B}_{2}, \ldots, \mathrm{B}_{\mathrm{n}}$, and assumptions[8,9]:

$$
K=\sum_{A_{\mathrm{i}} \cap B_{\mathrm{j}}} \mathrm{m}_{1}\left(A_{1}\right) \mathrm{m}_{2}\left(B_{2}\right)<1
$$

Where: $\mathrm{K}$ represents the degree of conflict between the two pieces of evidence. When $\mathrm{K}=1$ when, for the whole conflict, this time D - S combination rule cannot be used; when $\mathrm{K}<1$, completely non-conflict, this time, $\mathrm{D}$ - $\mathrm{S}$ can be used in combination rule. The probability assignment function $\mathrm{m}$ : $2^{\Theta} \rightarrow[0,1]$ for basic probabilities for all basic probability assignment of non-empty set A proposition A has the distribution function (trust in Proposition A's):

$$
m(A)=\frac{\sum_{A_{i} \cap B_{\mathrm{j}}} \mathrm{m}_{1}\left(A_{1}\right) \mathrm{m}_{2}\left(B_{2}\right)<1}{1-K}
$$

The promotion of the above twenty-two fusion rules to more evidence combination, the combined effects of a plurality of functions corresponding to the confidence level of the results can also function with a plurality of straight-confidence and said: 


$$
m(A)=\frac{\sum_{A_{\mathrm{i}} \cap B_{\mathrm{j}}=A} \mathrm{~m}_{1}\left(A_{\mathrm{i}}\right) \ldots \mathrm{m}_{\mathrm{n}}\left(A_{j}\right)}{1-\sum_{A_{\mathrm{i}} \cap B_{\mathrm{j}}=A} \mathrm{~m}_{1}\left(A_{\mathrm{i}}\right) \ldots \mathrm{m}_{\mathrm{n}}\left(A_{j}\right)}
$$

D-S Evidence Theory in the key is how to construct the basic probability of each focal element, the output of the neural network and fuzzy comprehensive evaluation as D-S evidence theory combination of evidence credibility. Network error between the actual output and the desired output of the fuzzy neural network is:

$$
E_{n}=\frac{1}{2} \sum_{j}\left(t_{n j}-y_{n j}\right)^{2}
$$

Where: $E_{n}$ is the $n$-th characterization network error vector; $t_{n j}$ for the $n$-th vector characterizing the $\mathrm{j}$-th output neuron expectations; $\mathrm{y}_{\mathrm{nj}}$ is $\mathrm{n}$ - $\mathrm{j}$ output neuron actual value of the $\mathrm{n}$ characterizing vector [10]. Basic probability values of fuzzy neural network diagnosis will normalize after the results are substituted into the formula (5), to give each sample $\mathrm{i}$-th failure mode $\mathrm{m}\left(\mathrm{A}_{\mathrm{i}}\right)$, the network error same treatment as D-S Evidence Theory the degree of uncertainty $\mathrm{m}(\Theta)$, construction of D-S evidence theoretical probability distribution value:

$$
\left\{\begin{array}{l}
m\left(A_{i}\right)=y\left(A_{i}\right) / S \\
\sum_{i=1} y\left(A_{i}\right)+E
\end{array}\right.
$$

Where: $A_{i}$ represents failure mode; $i=1,2, \ldots, n ; y(A i)$ fuzzy neural network diagnostic results. By D-S Evidence Theory method multiple fuzzy neural network diagnostic results in the decision-making level fusion, thereby obtaining fully reflect the equipment running diagnostic results.

\section{Simulation and Analysis}

In this paper, CNC milling machine (DX-600B) mechanical failure data as an example, in this way only the vibration signal and the accuracy of two road surface judgment result information fusion. Select E1 spindle fever, intermittent pauses when E2 spindle heavy cutting; E3 spindle work faint noise, E4 machining accuracy drops (surface morphology of a certain law), E5 machining accuracy drops (surface morphology no significant law) as the fault characteristic semaphores, forming a sample by normalization process after analysis, detection database. Because each input networks often have a different meaning and a different physical dimension, we need to enter sample normalized, normalized formula is as follows:

$$
T=X-X_{\min } / X_{\max }-X_{\min }
$$

Where: $X$ is the original data; $X_{\max }$ and $X_{\min }$ to the maximum and minimum values of the original data; $T$ is the transformed data, ie, the target data; article 100 select group of samples from the instance data, the sample group of 10 after normalization which data shown in table 1.The main fault is defined 5 types: $A_{1}$ spindle bearing preload is too large, $A_{2}$ spindle bearing damage, $A_{3}$ spindle motor spindle connected with the too loose, $\mathrm{A}_{4}$ spindle assembly balancing bad, $\mathrm{A}_{5}$ spindle drive gear wear. Establish sample database. The vibration sensor data, the accuracy of detecting surface data into the fuzzy neural network is trained, expected output and simulation results are shown in Table 2 and Table 3 , using the formula (1) method provides the basic structure of the probability of each fault in Table 4.

Similarly, the surface accuracy of the test data into the fuzzy neural network training simulation, and the results are shown in Table 5, and then construct the basic probability assignment (2). The results are shown in Table 6. Finally, more than two sub-diagnosis decision level fusion, coming in Tables 4 and 6 are fused to form a fusion diagnostic model based on Decision Level D-S evidence theory fusion results shown in Table 7, Table 4, Table 6 and Table 7 comparison, can be found after the fusion substantially improved diagnostic confidence in decision-making. 
Table 1 One of a sample treated normalized

\begin{tabular}{|l|l|l|l|l|l|l|}
\hline No. & $\mathrm{E}_{1}$ & $\mathrm{E}_{2}$ & $\mathrm{E}_{3}$ & $\mathrm{E}_{4}$ & $\mathrm{E}_{5}$ & Fault type \\
\hline 1 & 0.923 & 0.125 & 0.453 & 0.101 & 0.012 & $\mathrm{~A}_{1}$ \\
\hline 2 & 0.754 & 0.012 & 0.723 & 0.231 & 0.841 & $\mathrm{~A}_{2}$ \\
\hline 3 & 0.120 & 0.964 & 0.012 & 0.023 & 0.120 & $\mathrm{~A}_{3}$ \\
\hline 4 & 0.623 & 0.121 & 0.823 & 0.786 & 0.453 & $\mathrm{~A}_{4}$ \\
\hline 5 & 0.726 & 0.102 & 0.789 & 0.862 & 0.354 & $\mathrm{~A}_{5}$ \\
\hline
\end{tabular}

Table 2 The desired output of the detection data

\begin{tabular}{|l|l|l|l|l|l|}
\hline Number of Groups & $\mathrm{F}_{1}$ & $\mathrm{~F}_{2}$ & $\mathrm{~F}_{3}$ & $\mathrm{~F}_{4}$ & $\mathrm{~F}_{5}$ \\
\hline Group 1 & 1 & 0 & 0 & 0 & 0 \\
\hline Group 2 & 0 & 1 & 0 & 0 & 0 \\
\hline Group 3 & 0 & 0 & 1 & 0 & 0 \\
\hline Group 4 & 0 & 0 & 0 & 1 & 0 \\
\hline Group 5 & 0 & 0 & 0 & 0 & 1 \\
\hline
\end{tabular}

Table 3 Vibration signal subsystem failure diagnosis model simulation results

\begin{tabular}{|l|l|l|l|l|l|}
\hline Number of Groups & $\mathrm{A}_{1}$ & $\mathrm{~A}_{2}$ & $\mathrm{~A}_{3}$ & $\mathrm{~A}_{4}$ & $\mathrm{~A}_{5}$ \\
\hline Group 1 & 0.901 & 0.245 & 0.365 & 0.256 & 0.284 \\
\hline Group 2 & 0.324 & 0.832 & 0.246 & 0.612 & 0.714 \\
\hline Group 3 & 0.123 & 0.264 & 0.812 & 0.142 & 0.231 \\
\hline Group 4 & 0.146 & 0.679 & 0.303 & 0.836 & 0.641 \\
\hline Group 5 & 0.234 & 0.756 & 0.230 & 0.664 & 0.862 \\
\hline
\end{tabular}

Table 4 Vibration signal output subsystem basic probability distribution obtained

\begin{tabular}{|l|l|l|l|l|l|l|}
\hline Number of Groups & $\mathrm{m}\left(\mathrm{A}_{1}\right)$ & $\mathrm{m}\left(\mathrm{A}_{2}\right)$ & $\mathrm{m}\left(\mathrm{A}_{3}\right)$ & $\mathrm{m}\left(\mathrm{A}_{4}\right)$ & $\mathrm{m}\left(\mathrm{A}_{5}\right)$ & $\mathrm{m}(\Theta)$ \\
\hline Group 1 & 0.902 & 0.244 & 0.366 & 0.255 & 0.282 & 0.012 \\
\hline Group 2 & 0.323 & 0.833 & 0.247 & 0.613 & 0.713 & 0.122 \\
\hline Group 3 & 0.124 & 0.265 & 0.813 & 0.143 & 0.232 & 0.031 \\
\hline Group 4 & 0.145 & 0.678 & 0.304 & 0.835 & 0.640 & 0.021 \\
\hline Group 5 & 0.236 & 0.757 & 0.231 & 0.665 & 0.863 & 0.012 \\
\hline
\end{tabular}

Table 5 Surface accuracy subsystem failure diagnosis

\begin{tabular}{|l|l|l|l|l|l|}
\hline Number of Groups & $\mathrm{A}_{1}$ & $\mathrm{~A}_{2}$ & $\mathrm{~A}_{3}$ & $\mathrm{~A}_{4}$ & $\mathrm{~A}_{5}$ \\
\hline Group 1 & 0.900 & 0.255 & 0.375 & 0.276 & 0.294 \\
\hline Group 2 & 0.324 & 0.822 & 0.256 & 0.622 & 0.724 \\
\hline Group 3 & 0.133 & 0.274 & 0.812 & 0.162 & 0.241 \\
\hline Group 4 & 0.146 & 0.679 & 0.303 & 0.836 & 0.651 \\
\hline Group 5 & 0.239 & 0.757 & 0.255 & 0.674 & 0.852 \\
\hline
\end{tabular}

Table 6 Surface accuracy probability distribution function subsystem configuration

\begin{tabular}{|l|l|l|l|l|l|l|}
\hline Number of Groups & $\mathrm{m}\left(\mathrm{A}_{1}\right)$ & $\mathrm{m}\left(\mathrm{A}_{2}\right)$ & $\mathrm{m}\left(\mathrm{A}_{3}\right)$ & $\mathrm{m}\left(\mathrm{A}_{4}\right)$ & $\mathrm{m}\left(\mathrm{A}_{5}\right)$ & $\mathrm{m}(\Theta)$ \\
\hline Group 1 & 0.902 & 0.256 & 0.373 & 0.279 & 0.295 & 0.010 \\
\hline Group 2 & 0.321 & 0.823 & 0.255 & 0.623 & 0.723 & 0.112 \\
\hline Group 3 & 0.132 & 0.273 & 0.813 & 0.163 & 0.243 & 0.041 \\
\hline Group 4 & 0.143 & 0.678 & 0.302 & 0.835 & 0.652 & 0.022 \\
\hline Group 5 & 0.238 & 0.756 & 0.256 & 0.672 & 0.853 & 0.014 \\
\hline
\end{tabular}


Table 7 Level fusion results of D-S evidence theory decision

\begin{tabular}{|l|l|l|l|l|l|l|}
\hline Number of Groups & $\mathrm{m}\left(\mathrm{A}_{1}\right)$ & $\mathrm{m}\left(\mathrm{A}_{2}\right)$ & $\mathrm{m}\left(\mathrm{A}_{3}\right)$ & $\mathrm{m}\left(\mathrm{A}_{4}\right)$ & $\mathrm{m}\left(\mathrm{A}_{5}\right)$ & $\mathrm{m}(\Theta)$ \\
\hline Group 1 & 0.982 & 0.056 & 0.073 & 0.079 & 0.129 & 0.001 \\
\hline Group 2 & 0.121 & 0.923 & 0.155 & 0.123 & 0.123 & 0.012 \\
\hline Group 3 & 0.132 & 0.073 & 0.933 & 0.163 & 0.043 & 0.012 \\
\hline Group 4 & 0.143 & 0.178 & 0.012 & 0.955 & 0.152 & 0.002 \\
\hline Group 5 & 0.038 & 0.156 & 0.056 & 0.172 & 0.953 & 0.004 \\
\hline
\end{tabular}

\section{Conclusion}

For high-speed milling machine (DX-600B) Early diagnosis of mechanical failure, for D - S Evidence Theory in the application of basic probability assignment is difficult to determine the problem, using a plurality of fuzzy neural network output subsystem constructed D-S Evidence Theory basic probability assignment. Simulation results show that: The diagnostic system can effectively solve the motorized spindle mechanical failure uncertainty and increased electrical spindle early mechanical failure diagnosis accuracy, the results more reliable.

\section{References}

[1] Wang Jiahai, Huang Jiangtao, Shen Bin, and so on. Research Status and Prospect of CNC machine tools Intelligent Fault Diagnosis [J]. Machinery manufacturing, 2014 (5): 30 - 32

[2] Yang Xiaobo, Lu Chen, Qin Juan. Bearing Fault Diagnosis of Motor [J]. Sensors and Microsystems. 2010 (07)

[3] $\mathrm{Hu}$ Shousong, HE Yaqun. Rough decision theory and applications [M]. Beijing: Beijing University of Aeronautics and Astronautics Press, 2006

[4] Sun Hua, Zhao Zhenfeng. State prediction algorithm for electronic equipment based on the rough set theory [J]. Ship Electronic Engineering, 2009, 29 (10): 159-162.

[5] Zhang Dongchu, PEI Xuming. Effects of machining processes on surface roughness and fatigue life [J]. China Mechanical Engineering, 2003, 14 (16): 1374-1377.

[6] Luo Zhi-ming, FENG Geng-bin. B Locomotive Rolling BP network fault diagnosis method [J]. Journal of the China Railway Science, 1998, 19 (4): 26--31.

[7] Chen C Z, Li Q, Song H Y. Wear fault diagnosis of machinery based on neural networks and gray relationships [J]. International Journal of Plant Engineering and Man Age, 2001, 6(3): 164--169.

[8] Tang Tian, Zhang Xiangli, and so on. Machine Tool Servo System Fault Based on LM-BP neural network diagnosis [J]. Guilin University of Electronic Technology, 2013 (3): 218-222.

[9] Wu Dongmei, Shao Jianping. Based on Machine Fault Diagnosis CNC ant colony algorithm and neural network [J]. Mechanical design and manufacturing, 2013 (1): 165--167.

[10]MO Qi, Information Fusion Fault Diagnosis of Rotating Machinery Application [J]. Manufacturing Automation, 2010, 32 (4): 76-77. 